\title{
Sistemas de manejo e parâmetros da matéria orgânica de um solo do cerrado baiano, Brasil
}

Este trabalho objetivou avaliar um Latossolo Vermelho-amarelo de textura Franca-arenosa no Oeste da Bahia, Brasil, cultivado com soja (S) sob plantio convencional (PC) e sistema plantio direto (SPD), utilizando como parâmetros, o Carbono Orgânico Total (COT), a Biomassa Microbiana do Solo (BMS), a Respiração Basal do Solo (RBS), o Quociente Microbiano (qMic), o Quociente Metabólico (qCO2), a Densidade do Solo (Ds) e os Estoques de Carbono (Est_C). O delineamento experimental foi de casualização por blocos com quatro repetições sendo os tratamentos T1: Soja sob Plantio Convencional (S_PC) e T2: Soja sob Sistema Plantio Direto (S SPD), tendo como referência, um Cerrado nativo (CN), de fitofisionomia 'Campo sujo', adjacente à área experimental. Amostras de solo foram coletadas nas camadas 0,00 a 0,05; 0,05 a 0,10 e 0,10 a 0,20 m. Os dados obtidos foram submetidos à ANOVA e as médias comparadas pelo teste de Duncan ao nível de significância de $p$ $=0,05$. Os resultados evidenciam que o SPD, em relação ao PC, promove os valores do COT, da BMS, do qMic e reduz os valores de qCO2 nas camadas do solo avaliado. Além disso, o SPD promoveu os valores dos Est C, contrastando com os valores observados no solo sob PC. Conclui-se que a SPD melhora a qualidade do solo em comparação ao PC, porque favorece os estoques de carbono.

\section{Management systems and parameters of soil organic matter of the savannah of Bahia, Brazil}

\begin{abstract}
The objective of this work was to evaluate a Red-Yellow Latosol of a sandy-loam texture in the West of Bahia, Brazil, cultivated with soybean (S) under conventional tillage (CT) and no-tillage system (NTS) using Total Organic Carbon (TOC), Soil Microbial Biomass Carbon (SMB-C), Soil Basal Respiration (SBR), Microbial quotient (Micq), Metabolic quotient (qCO2), Bulk density (Bd) and Carbon Stocks (C_St).The experimental design was a randomized block with four replications: T1 treatments: Soybean (S) under Conventional tillage (S_CT) and T2: Soybean under No-Tillage System (S_NTS), having as reference, a Native Savannah (NS), of phytophysiognomy 'Dirty Field' adjacent to the experimental area. Soil samples were collected in the layers 0.00 to $0.05 ; 0.05$ to 0.10 and 0.10 to $0.20 \mathrm{~m}$. The data were submitted to ANOVA and the means were compared by the Duncan test at the significance level of $p=0.05$. The results show that the NTS, in relation to the $\mathrm{CT}$, promotes the values of TOC, SMB-C, Micq and reduces the values of qCO2 in the layers of the evaluated soil. In addition, the NTS promoted the values of the C_St, contrasting with the values observed in the soil under CT. It is concluded that SPD improves soil quality compared to CT. The NTS promotes bioindicators of soil quality, especially carbon stocks.
\end{abstract}

Keywords: Brazilian Savannah; Carbon Stock; Soil Microbial Biomass; Agricultural and Environmental Sustainability; Soil Organic Matter.

\section{Topic: Uso de Recursos Naturais}

Reviewed anonymously in the process of blind peer.
Received: $12 / 04 / 2019$

Approved: 27/05/2019
Francisco Rubens Feitosa Júnior (iD

Universidade Federal do Oeste da Bahia, Brasil http://lattes.cnpq.br/1603533849627421 http://orcid.org/0000-0001-7793-2138 rubensjr21@hotmail.com

Diony Alves Reis (DD)

Universidade Federal do Oeste da Bahia, Brasil http://lattes.cnpq.br/5707542223794836

http://orcid.org/0000-0003-4270-2978 dionyodin@gmail.com

\section{Roberto Baggatini Portella (iD)}

Universidade Federal do Oeste da Bahia, Brasil http://lattes.cnpq.br/3464794820553147

http://orcid.org/0000-0003-4236-3128

roberto.portella@ufob.edu.br

\author{
Jackson Roberto de Souza Santos (iD) \\ Universidade Federal do Oeste da Bahia, Brasil \\ http://lattes.cnpq.br/0236039035829068 \\ http://orcid.org/0000-0002-2773-6873 \\ santos.jrs@gmail.com \\ Fabiano José Perina \\ Embrapa Algodão, Brasil \\ http://lattes.cnpq.br/2414090933616921 \\ http://orcid.org/0000-0003-4049-3383 \\ fabiano.perina@embrapa.br \\ Julio Cesar Bogiani \\ Embrapa Territorial, Brasil \\ http://lattes.cnpq.br/2124564195422694 \\ http://orcid.org/0000-0002-3357-7944 \\ julio.bogiani@embrapa.br
}

\section{Referencing this:}

FEITOSA JÚNIOR, F. R.; REIS, D. A.; PORTELLA, R. B.; SANTOS, J. R. S.; PERINA, F. J.; BOGIANI, J. C.. Sistemas de manejo e parâmetros da matéria orgânica de um solo do cerrado baiano, Brasil. Revista Ibero Americana de Ciências Ambientais, v.10, n.3, p.298-312, 2019. DOI: http://doi.org/10.6008/CBPC2179-6858.2019.003.0025 


\section{INTRODUÇÃO}

Entre as culturas de destaque no Brasil, a soja representa $51 \%$ da produção nacional de grãos. Na safra 2018/19 a produção de grãos nacional foi estimada em 240,7 milhões de toneladas, e área plantada de 62,9 milhões de hectares. A produção de soja foi de 115 milhões de toneladas (t), um decréscimo de 3,6\% em relação a 2017/18, e a estimativa de produtividade em 2019 foi de $3.207 \mathrm{~kg} \mathrm{ha}^{-1}$, sendo 717,4 mil hectares de área plantada (CONAB, 2019). Na Bahia, na safra de 2018/19, foram plantadas cerca de 1.580 mil ha de soja, 1,2\% menor que a área plantada em 2017/18. A produção atual de 5.309 mil $t$, foi $16 \%$ menor que a anterior, enquanto a produtividade da safra atual, estimada em $3.360 \mathrm{~kg} \mathrm{ha}^{-1}$, representa um decréscimo de aproximadamente 15\% em relação à produtividade de 2017/18 (CONAB, 2019).

Para a o Ministério da Agricultura, Pecuária e Abastecimento (MAPA, 2017), a região que recobre parte dos territórios dos estados do Maranhão, Tocantins, Piauí e Bahia, (MATOPIBA) é uma cadeia produtiva complexa, composta por um conjunto de 337 municípios, somando um total de 73 milhões de ha, 324.000 propriedades agrícolas, 46 unidades de Conservação Ambiental, 35 Áreas indígenas e 781 Assentamentos de Reforma Agrária.

O MATOPIBA vem se destacando desde 1990, quando saiu de 260 mil t de soja para 2,2 milhões; atingindo 7 milhões de t em 2016 e na safra de 2017/18 alcançou a produção de 9 milhões de t de soja (AIBA, 2018). Esta região agrícola foi responsável, na safra de $2017 / 18$, por $14 \%$ da produção brasileira, que correspondeu a uma produção de 16,4 milhões de t de soja (CONAB, 2019), sobretudo devido ao aumento de área plantada em comparação a 2017/18 (CONAB, 2019).

Em decorrência da expansão do agronegócio, a importância de se avaliar os sistemas de manejo do solo torna-se fundamental. O Plantio Convencional (PC), preconiza o revolvimento frequente do solo por meio das práticas de aração e gradagem (SEGUY et al., 2012), sem manutenção dos resíduos das culturas, favorecendo a exposição do solo às intempéries climáticas e reduções nos teores de matéria orgânica e carbono no solo (LEPSCH, 2010). Por outro lado, no Sistema de Plantio Direto (SPD), o solo passa a ter uma cobertura permanente, a semeadura é localizada, sem revolvimento, realizando rotação de culturas (NUNES, 2014) garantindo a proteção, a conservação e promoção dos estoques de carbono (ALVAREZ et al., 2014) e a qualidade do solo (REIS et al., 2019; DADALTO et al., 2015).

$\mathrm{O}$ uso dos solos, sobretudo em extensas áreas agrícolas, como as observadas no Cerrado, tem causado modificações nos teores de matéria orgânica, na biomassa e na atividade microbiana do solo, evidenciando processo de degradação, que podem ser mitigados através de manejos que possibilitem incrementos de matéria orgânica e carbono no solo (ROSSI et al., 2011). Nesse sentido, o preparo intensivo com o uso de arados, grades e subsoladores são os principais responsáveis pela degradação acelerada do solo (FERREIRA et al., 2010; ROMERO et al., 2016).

A dinâmica da matéria orgânica influencia os principais processos químicos, físicos e biológicos nos solos, determinando a sua fertilidade. O solo é o habitat para vários microrganismos que utilizam a matéria orgânica do solo (MOS) como fonte de alimentos, além disso, a MOS tem sido considerada como o melhor 
indicador de qualidade do solo devido à sua influência na estruturação e agregação do solo (ROSSI et al., 2011; SALTON et al., 2011; SILVA JÚNIOR, 2016).

A quantificação da MOS tem auxiliado no monitoramento da qualidade do solo e seu incremento tem sido utilizado como ferramenta para a recuperação de solos degradados, melhorando sua estrutura, reduzindo a densidade do solo, a resistência do solo à penetração de raízes à medida que a estabilidade de agregados, a porosidade e retenção de água são promovidos, que por sua vez, estas alterações favorecem o crescimento e o desenvolvimento das plantas (RESENDE et al., 2012).

A Biomassa Microbiana do solo (BMS) se destaca como indicador de qualidade do solo devido à sensibilidade que os microrganismos apresentam frente às alterações promovidas pelos sistemas de manejo. Além disso, a atividade microbiana é afetada pela umidade, temperatura, estrutura do solo, textura e disponibilidade de matéria orgânica, consequentemente, pelas práticas agrícolas (SILVA et al., 2010).

A BMS é um indicador biológico de qualidade do solo, que se relaciona com a manutenção, a produtividade dos agroecossistemas, com processos e atividades mediadas pelos microrganismos. Ela representa a fração viva da matéria orgânica, sendo constituída por fungos, bactérias e actinomicetos que desempenham processos fundamentais para a manutenção do ecossistema, porém são sensíveis às modificações no ambiente (CONTI et al., 2012; FREITAS et al., 2018).

Em solos sob PC, onde o revolvimento causa a quebra dos agregados, gera maior contato entre os resíduos orgânicos e o solo, incita a microbiota a degradar a MOS mais rapidamente (LISBOA et al., 2012). A atividade microbiana associa-se à qualidade do solo, sendo possível, através das análises de BMS e da relação com a respiração basal do solo (RBS) obter indicadores que caracterizam a qualidade e o estado de degradação do solo (LOURENTE et al., 2011).

A RBS é definida como a soma de todas as funções metabólicas nas quais o $\mathrm{CO}_{2}$ é produzido, ou seja, a RBS expressa a produção de $\mathrm{CO}_{2}$ no solo advinda da atividade respiratória de microrganismos (POLLI et al., 2005; LOURENTE et al., 2011; ELEFTHERIADIS et al., 2014; ZHOU et al., 2017; GOENSTER et al., 2017). A interpretação dos dados da RBS deve levar em consideração o uso ou as modificações no solo, pois, segundo Silva et al. (2007) e Zhou et al. (2017) o incremento na atividade respiratória pode ser desencadeado tanto pela alta produtividade de um determinado ecossistema, quanto pelo estresse advindo de distúrbios ambientais.

O quociente metabólico $\left(\mathrm{qCO}_{2}\right)$ é a razão entre a RBS e a BMS por unidade de tempo, e fornece informações sobre a atividade microbiológica do solo, demonstrando a taxa de respiração microbiana (ALVES, 2011; LOURENTE et al., 2011; ELEFTHERIADIS et al., 2014; ZHOU et al., 2017; GOENSTER et al., 2017) e tem sido utilizado para avaliar a eficiência do uso de substrato pelos microrganismos do solo.

O quociente microbiano (qMic) fornece indicações sobre a qualidade da matéria orgânica, sendo expresso pela relação entre a BMS e o carbono orgânico total (COT) (SILVA et al., 2010; DUARTE et al., 2014). Em condições estressantes para os microrganismos a capacidade de utilização do $C$ é menor, conduzindo ao decréscimo do qMic, enquanto com a adição de matéria orgânica em quantidade e qualidade ou com o término de uma situação de estresse, ocorre um incremento na BMS, assim como no qMic, ainda que os 
teores de COT do solo permaneçam praticamente inalterados (SILVA et al., 2010).

O qMic representa o percentual de reserva do COT no solo e em solos de baixa atividade microbiana apresentam baixos valores, indicando menor reserva de compostos orgânicos (CARNEIRO, 2009; SILVA et al., 2010). Valores elevados de qMic demonstram maior quantidade de matéria orgânica e promoção da atividade microbiana do solo.

No Brasil, os trabalhos pioneiros afirmam que o balanço entre as adições e perdas de Carbono (C), em um estado de equilíbrio dinâmico, onde praticamente não existe variação no teor de $\mathrm{C}$ orgânico com o tempo, é definido como estoque de C no solo (Est_C), porém, Martín et al. (2016) afirmam que as atividades humanas alteram o ciclo de carbono adicionando mais $\mathrm{CO}_{2}$ à atmosfera e influenciando as funções do solo, sobretudo aquelas relacionadas ao sequestro de carbono.

Conforme Houghton et al. (2001), o C se acumula na atmosfera a uma taxa de 3,5 Pg C ano-1 (Pg = $10^{15} \mathrm{~g}$ ), enquanto os vegetais armazenam em torno de $550 \mathrm{Pg}$ de $\mathrm{C}$, além disso, o $\mathrm{C}$ armazenado no primeiro metro de solos nos ecossistemas terrestres é de cerca de 2 a 4 vezes a quantidade encontrada na vegetação (1500 Pg C). O preparo do solo, o uso do fogo, mudanças no uso da terra como o desmatamento, implementação de pastagens e adoção de sistemas agrícolas intensivos ou com métodos desfavoráveis à manutenção dos atributos do solo, são apontados como causas recorrentes da alteração dos Est_C no solo Martín et al. (2016).

Neste sentido, este trabalho objetiva avaliar a qualidade de um Latossolo Vermelho amarelo distrófico, cultivado com soja sob plantio convencional e sistema plantio direto no Cerrado baiano, utilizando como bioindicadores de qualidade, o Carbono Orgânico Total (COT), o Carbono da Biomassa microbiana (CBMS), a Respiração Basal do Solo (RBS), o Quociente Microbiano (qMic), o Quociente Metabólico ( $\left(\mathrm{CO}_{2}\right.$ ), a Densidade do Solo (Ds) e os estoques de carbono (Est_C).

\section{MATERIAIS E MÉTODOS}

\section{Área de estudo}

O estudo foi realizado no município de Luís Eduardo Magalhães, Bahia, Brasil, em área experimental pertence à Fundação de Apoio à Pesquisa e Desenvolvimento do Oeste Baiano (Fundação BA), sendo as coordenadas geográficas do experimento, $12^{\circ} 5^{\prime} 36.52^{\prime \prime} \mathrm{S}$ de latitude Sul e $45^{\circ} 42^{\prime} 40.30^{\prime \prime} \mathrm{O}$ de longitude Oeste.

A área está inserida no bioma Cerrado, que apresenta duas estações climáticas distintas, uma chuvosa, entre os meses de outubro e março, com precipitação média de $1500 \mathrm{~mm}$; e uma estação seca, sem precipitações nos demais meses. A temperatura média anual gira em torno de $22^{\circ}$ a $27^{\circ} \mathrm{C}$ (MALHEIROS, 2016), sendo o clima classificado como do tipo Aw, conforme a classificação climática de Köppen-Geiger (ALVARES et al., 2013).

Anterior ao plantio, na área foi realizada a calagem, a subsolagem e a correção das características químicas do solo. $\mathrm{O}$ solo estudado é um Latossolo Vermelho Amarelo distrófico, com textura franca-arenosa (804 $\mathrm{g} \mathrm{kg}^{-1}$ de areia, $74 \mathrm{~g} \mathrm{~kg}^{-1}$ de silte, e $125 \mathrm{~g} \mathrm{~kg}^{-1}$ de argila) até a profundidade de 0,20 m, apresentando um 
relevo plano.

O delineamento experimental foi o de casualização por blocos com quatro repetições sendo os tratamentos: T1: Soja (S) sob Plantio Convencional (S_PC); T2: S sob Sistema Plantio Direto (S_SPD), tendo como referência uma área de Cerrado Nativo (CN), não antropizada e de fitofisionomia de Campo Sujo, adjacente ao experimento. Além disso, o histórico de cultivo da área, nos últimos cinco anos, é apresentado na Quadro 1.

Quadro 1: Sucessão cultural agrícola em um Latossolo cultivado com soja sob plantio convencional (S_PC) e sistema plantio direto (S_SPD).

\begin{tabular}{|l|l|l|l|l|l|l|}
\hline Tratamentos* & $2012 / 13$ & $2013 / 14$ & $2014 / 15$ & $2015 / 16$ & $2016 / 17$ & $2017 / 18$ \\
\hline S_PC & Soja & Soja & Soja & Soja & Soja & Soja \\
\hline S_SPD & Milho + Braquiária & Algodão & Soja Crotalária & Milho + Braquiária & Algodão & Soja Crotalária \\
\hline
\end{tabular}

*S_PC: Soja sob Plantio Convencional; S_SPD: Soja sob Sistema Plantio Direto, em consórcio com Crotalária sucedendo Milho, Braquiária e Algodão.

\section{Amostragem do solo}

As amostras do solo com estrutura não preservada e preservada foram coletadas nas camadas 0,00 a 0,05; de 0,05 a 0,10 e 0,10 a 0,20 m. Para a coleta das amostras de solo com estrutura não preservada uma pá de corte foi utilizada, sendo coletadas 24 amostras (1 amostra x 3 camadas de solo $\times 4$ blocos $\times 2$ tratamentos), que foram utilizadas para determinação das frações granulométricas, do carbono orgânico total (COT), do carbono da biomassa microbiana do solo (C-BMS) e da respiração basal do solo (RBS). O solo com estrutura preservada foi coletado em anéis volumétricos de $4,8 \mathrm{~cm}$ de diâmetro por $4,5 \mathrm{~cm}$ de altura totalizando 72 amostras ( 3 anéis por camada $\times 3$ camadas de solo $\times 4$ blocos $\times 2$ tratamentos), que foram utilizadas para a determinação da densidade do solo (Ds).

\section{Análises do solo}

As frações granulométricas foram determinadas pelo Método da Pipeta, utilizando-se $10 \mathrm{~g}$ de terra fina seca em estufa (TFSE) que foram suspensos em provetas de $1000 \mathrm{ml}$ utilizando $\mathrm{NaOH}$ a $1 \mathrm{~N}$, conforme Teixeira et al. (2017). O carbono orgânico total (COT) foi determinado por meio da oxidação da matéria orgânica via $\mathrm{K}_{2} \mathrm{Cr}_{2} \mathrm{O}_{7}$ a 0,0667 mol L-1 em meio sulfúrico, sendo posteriormente titulado com solução padrão de $\mathrm{Fe}\left(\mathrm{NH}_{4}\right)_{2}\left(\mathrm{SO}_{4}\right) 2.6 \mathrm{H}_{2} \mathrm{O}$ a $0,1 \mathrm{~mol} \mathrm{~L}^{-1}$ (TEIXEIRA et al., 2017).

A determinação do carbono da biomassa microbiana do solo (C-BMS), foi realizada pelo método de fumigação e extração (VANCE et al., 1987), utilizando uma amostra de $10 \mathrm{~g}$ de solo, e para a fumigação, 1,0 $\mathrm{mL}$ de $\mathrm{CHCl}_{3}$ foi aplicado diretamente sobre a amostra que ficou incubada por 24 horas em recipiente fechado em ambiente sem iluminação (POLLI et al., 2008). Para extração utilizou-se $25 \mathrm{~mL}$ de $\mathrm{K}_{2} \mathrm{SO}_{4}$ a $0,5 \mathrm{~mol} \mathrm{~L}^{-1}$ e 1,0 $\mathrm{mL}$ de $\mathrm{K}_{2} \mathrm{Cr}_{2} \mathrm{O}_{7}$ sendo titulada com $\mathrm{Fe}\left(\mathrm{NH}_{4}\right)_{2}\left(\mathrm{SO}_{4}\right) 2.6 \mathrm{H}_{2} \mathrm{O}$ a $0,33 \mathrm{~mol} \mathrm{~L}-1$ tendo como indicador $\left(\mathrm{C}_{6} \mathrm{H}_{5}\right)_{2} \mathrm{NH}$. O cálculo do C-BMS (mg kg-1 de BMS no solo) foi realizado por meio das Equações:

$$
\mathrm{BMS}=\mathrm{FC}^{*} \mathrm{kc}^{-1} \quad \text { Eq. } 1
$$

Onde:

$$
\mathrm{FC}=\mathrm{CSF}-\mathrm{CSNF} \quad \text { Eq. } 2
$$


Sendo CSF o teor de C extraído do solo fumigado, CSNF o teor de C extraído do solo não fumigado e kc - fator de correção, utilizando o valor de 0,33, conforme sugerido em Silva et al. (2007).

$$
\text { CSF ou CSFN }=\frac{\left(V_{b}-V_{a}\right) \cdot M \cdot 0 \cdot 003 \cdot V_{1} \cdot 10^{6}}{P_{S} \cdot V_{2}} \quad \text { Eq. } 3
$$

Em que $\mathrm{Vb}$ - volume $(\mathrm{mL})$ de $\left(\mathrm{NH}_{4}\right)_{2} \mathrm{Fe}\left(\mathrm{SO}_{4}\right)_{2} .6 \mathrm{H}_{2} \mathrm{O}$ gasto na titulação da solução do ensaio em branco; $\mathrm{Va}$ - volume $(\mathrm{mL})$ de $\left(\mathrm{NH}_{4}\right)_{2} \mathrm{Fe}(\mathrm{SO} 4) 2.6 \mathrm{H}_{2} \mathrm{O}$ gasto na titulação da solução do ensaio com a amostra; $\mathrm{M}$ - Molaridade exata do sulfato ferroso amoniacal (0.033); 0,003 - miliequivalente do carbono; $\mathrm{V}_{1}$ - volume do extrator $\left(\mathrm{K}_{2} \mathrm{SO}_{4}\right)$ utilizado; $\mathrm{V}_{2}$ - alíquota pipetada do extrato para a titulação; Ps (g) - massa de solo seco.

A respiração basal do solo (RBS), foi determinada conforme o procedimento descrito por Jenkinson et al., (1976), utilizando-se $100 \mathrm{~g}$ de solo incubadas hermeticamente em frascos de vidro na presença de 10 $\mathrm{mL}$ de $\mathrm{NaOH}$ a $0,5 \mathrm{~mol} \mathrm{~L}^{-1}$ por 7 dias. $\mathrm{O}$ cálculo da RBS ( $\mathrm{mg} \mathrm{kg}^{-1} \mathrm{~h}^{-1} \mathrm{C}-\mathrm{CO}_{2}$ no solo) foi realizado pela Equação 4:

$$
\mathrm{RBS}=\frac{\left(\mathrm{V}_{\mathrm{b}}-\mathrm{V}_{\mathrm{a}}\right) \mathrm{CHCl} * 6 * 1000}{\mathrm{M}_{\mathrm{s}} * \mathrm{~T}} \quad \text { Eq. } 4
$$

Em que, $\mathrm{Vb}$ - representa o volume de $\mathrm{HCl}(\mathrm{mL})$ gasto na titulação do $\mathrm{NaOH}$ do ensaio em branco; $\mathrm{Va}$ - volume de $\mathrm{HCl}$ $(\mathrm{mL})$ gasto na titulação do $\mathrm{NaOH}$ do ensaio com a amostra, $\mathrm{CHCl}$, concentração do $\mathrm{HCl}\left(\mathrm{mol} \mathrm{L}^{-1}\right)$, $\mathrm{Ms}$ - massa de solo incubado seco (g); e T - tempo de incubação $(\mathrm{h})$.

Posterior às determinações, o quociente microbiano ( $q \mathrm{Mic}$ ) foi calculado pela relação entre a BMS e o COT, expresso em porcentagem, conforme a Equação 5 (INSAM et al., 1988)

$$
\mathrm{qMic}=\left(\frac{\mathrm{BMS}}{\mathrm{COT}}\right) \times 100 \quad \text { Eq. } 5
$$

Enquanto $\mathrm{O} \mathrm{qCO}_{2}$ foi calculado pela razão entre a respiração RBS e BMS conforme descrito por Anderson et al. (1993) em $\left(\mu \mathrm{kg}^{-1} \mathrm{~h}^{-1}\right)$, por meio da Equação 6 .

$$
\mathrm{qCO}^{2}=\frac{\mathrm{RBS}}{\mathrm{BMS}}, \mathrm{mg} \mathrm{C}-\mathrm{CO}_{2} \mathrm{~g}^{-1} \mathrm{C}-\mathrm{BMS} \mathrm{h}^{-1} \quad \text { Eq. } 6
$$

Os estoques de C (Est_C) foram calculados pela massa equivalente (SISTI et al., 2004), utilizando-se a densidade média do solo em cada camada e tratamento, tendo o solo sob Cerrado Nativo (CN) não cultivado, como referência, utilizando-se a Equação 7.

$$
\mathrm{Cs}=\sum_{\mathrm{i}=1}^{\mathrm{n}-1} \mathrm{C}_{\mathrm{ti}}+\left[\mathrm{M}_{\mathrm{tn}}-\left(\sum_{\mathrm{i}=1}^{\mathrm{n}} \mathrm{M}_{\mathrm{Ti}}-\sum_{\mathrm{i}=1}^{\mathrm{n}} \mathrm{MS}_{\mathrm{i}}\right)\right] \mathrm{C}_{\mathrm{tn}}
$$

Onde $\mathrm{Cs}$, corresponde ao estoque de $\mathrm{C}$ corrigido em função da massa de solo da área de referência $\left(\mathrm{Mg} \mathrm{Cha}{ }^{-1}\right)$;

$\sum_{i=1}^{n-1} C_{T i}$ é a soma do carbono orgânico total $\left(\mathrm{Mg} \mathrm{ha}^{-1}\right)$ da camada superficial à penúltima camada $(\mathrm{n}-1)$ do tratamento avaliado; $\mathrm{M}_{\mathrm{tn}}$, massa do solo da última camada amostrada no tratamento ( $\mathrm{Mg}$ ha-1 ${ }^{-1}$; $\sum_{i=1}^{n} M_{T i}$ é a soma da massa de solo $\left(\mathrm{Mg} \mathrm{ha}^{-1}\right)$ na camada superficial (1) até a camada mais profunda (n) no tratamento avaliado; $\sum_{i=1}^{n} M S_{i}$ somatório da massa total do solo amostrado na área de referência $\left(\mathrm{Mg} \mathrm{ha}^{-1}\right)$ e $\mathrm{C}_{\mathrm{tn}}$, teor de $\mathrm{C}$ do solo na última camada amostrada ( $\mathrm{Mg} \mathrm{C} \mathrm{Mg}^{-1}$ de solo).

Antes da correção pela massa de solo, os estoques de C de cada camada, em todas as áreas estudadas, foram calculados pela Equação 8 (VELDKAMP, 1994).

$$
\text { Est }_{\mathrm{C}}=\frac{(\text { COT x Ds x e) }}{10}
$$

Em que Est_C é o estoque de COT total em determinada profundidade ( $M g$ ha-1 $)$; COT, é o teor de Corgânico total na profundidade amostrada $\left(\mathrm{g} \mathrm{kg}^{-1}\right)$; Ds, é a densidade do solo da profundidade avaliada $\left(\mathrm{Mg} \mathrm{m}^{-3}\right)$, e e representa a espessura da camada considerada $(\mathrm{cm})$.

As amostras com estrutura preservada foram secas em estufa a $105^{\circ} \mathrm{C}$, até estabelecimento de massa 
constante, obtendo-se a Densidade do Solo (Ds) (TEIXEIRA et al., 2017).

\section{Procedimentos estatísticos}

A normalidade dos dados foi verificada pelo teste de Shapiro-Wilk (W) ( $n \leq 200)$ (RAZALLI et al., 2011). Valores discrepantes (outliers) foram identificados através das medidas do limite inferior (LI) e do limite superior (LS), considerando o primeiro quartil (Q1), o terceiro quartil (Q3) e 1,5 amplitude interquartílica e foram substituídos pela média dos valores imediatamente superior e inferior.

Os tratamentos foram comparados através da diferença percentual do valor do parâmetro no tratamento, evidenciado seu incremento (+) ou diminuição (-) em relação ao CN ( $\Delta$ ref, \%) e, excluindo-se o $\mathrm{CN}$, os tratamentos foram submetidos à análise de variância (Anova) e as médias comparadas pelo teste de Duncan. Além disso, as relações entre as variáveis serão avaliadas por meio do coeficiente de correlação de Pearson (r) ao nível de significância de 5\%, utilizando-se o software Statistical Analyses System Institute (SAS, 1999).

\section{RESULTADOS E DISCUSSÃO}

Na Tabela 1 são apresentas as médias e os desvios-padrão do COT e C-BMS do solo sob PC e SPD em comparação com um Cerrado Nativo (CN).

Tabela 1: Média e desvio-padrão de Carbono orgânico total (COT) e Biomassa Microbiana do Solo (C-BMS) de um Latossolo cultivado com Soja sob plantio convencional (S_PC) e sistema plantio direto (S_SPD) tendo um Cerrado nativo de fitofisionomia de campo sujo ( $\Delta$ ref, \%) como referência no Oeste da Bahia, Brasil.

\begin{tabular}{|c|c|c|c|c|c|c|}
\hline \multirow[t]{3}{*}{ Tratamento* } & COT & $\Delta$ ref & C-BMS & $\Delta$ ref & RBS & $\Delta$ ref \\
\hline & $\mathrm{g} \mathrm{kg}^{-1}$ & $\%$ & $\mathrm{mg} \mathrm{C} \mathrm{kg}^{-1}$ & $\%$ & $\mu \mathrm{g} \mathrm{kg}^{-1} \mathrm{~h}^{-1}$ & $\%$ \\
\hline & \multicolumn{6}{|l|}{0,00 a $0,05 \mathrm{~m}$} \\
\hline S_PC & $3,66 \pm 0,68 b$ & -43 & $164,55 \pm 42,66 b$ & -81 & $166,84 \pm 22,82 \mathrm{a}$ & 18 \\
\hline S_SPD & $7,41 \pm 1,89 a$ & 14 & $538,09 \pm 125,35 a$ & -38 & $94,09 \pm 10,08 b$ & -34 \\
\hline \multirow[t]{2}{*}{$\mathrm{CN}$} & 6,49 & 100 & 867,6 & 100 & 141,64 & 100 \\
\hline & \multicolumn{6}{|l|}{0,05 a $0,10 \mathrm{~m}$} \\
\hline S_PC & $3,91 \pm 0,48 b$ & -18 & $159,91 \pm 27,36 \mathrm{~b}$ & -83 & $162,49 \pm 18,02 a$ & 17 \\
\hline S_SPD & $5,11 \pm 0,60 \mathrm{a}$ & 6 & $513,71 \pm 119,31 \mathrm{a}$ & -47 & $93,18 \pm 25,80 \mathrm{~b}$ & -33 \\
\hline \multirow[t]{2}{*}{$\mathrm{CN}$} & 4,81 & 100 & 965,45 & 100 & 138,99 & 100 \\
\hline & \multicolumn{6}{|l|}{0,10 a $0,20 \mathrm{~m}$} \\
\hline S_PC & $2,76 \pm 1,13 b$ & -37 & $179,20 \pm 24.91 \mathrm{~b}$ & -81 & $72,48 \pm 22,46 \mathrm{a}$ & -40 \\
\hline S_SPD & $4,42 \pm 0,56 \mathrm{a}$ & $-0,6$ & $495,95 \pm 59,71 \mathrm{a}$ & -47 & $80,50 \pm 17,42 \mathrm{a}$ & -33 \\
\hline $\mathrm{CN}$ & 4,45 & 100 & 931,68 & 100 & 120,06 & 100 \\
\hline
\end{tabular}

*Soja em Plantio Convencional (S_PC); Soja em consórcio com Crotalária sucedendo Milho, Braquiária e Algodão (S_SPD); Cerrado nativo de campo sujo (CN). Médias seguidas pela mesma letra minúscula nas colunas, dentro de cada parâmetro e camada de solo avaliada não diferem pelo teste de Duncan a 5 \%.

O valor de COT observado no solo sob PC, na camada de 0,00 a $0,05 \mathrm{~m}$, foi $43 \%$ menor do que o observado no CN, contrastando o com o SPD, que evidenciou valor de COT 14\% maior que o observado na área de referência (Tabela 1). Na camada de 0,05 a 0,10 m o valor de COT no solo sob PC foi $18 \%$ menor que o verificado no CN, enquanto em SPD foi $6 \%$ maior e na camada de 0,10 a $0,20 \mathrm{~m}$ o COT em PC foi $37 \%$ menor do que no $\mathrm{CN}$, enquanto o valor no solo sob SPD foi $\sim 1 \%$ menor.

No trabalho de Jakelaitis et al. (2008) foi avaliada a qualidade do solo sob vegetação nativa, pastagem e áreas cultivadas, sendo observado que os teores de COT diminuíram em função do uso do solo, 
demonstrando que o conteúdo de carbono é influenciado pelo manejo (GUARESCHI et al., 2012; KOPITTKE et al., 2017; TANG et al., 2019). Além disso, Costa Junior et al. (2011) encontraram em áreas de Cerradão, em comparação a áreas de lavoura seguida de pastagem de 29 anos, o maior conteúdo de COT na camada de 0,00 a 0,20 m na área não antropizadas. De forma semelhante, Gazolla et al. (2015), também verificaram que solos manejados têm menor conteúdo de $\mathrm{C}$ quando comparados com áreas não manejadas.

Os valores de COT obtidos no Cerrado Campo Sujo avaliados nas diferentes camadas, respectivamente, foram 6,49; 4,81 e 4,45 $\mathrm{g} \mathrm{kg}^{-1}$ (Tabela 1) expressivamente menores do que os obtidos por Costa Junior et al. (2011) que encontraram num Latossolo de textura argilosa sob Cerradão, respectivamente, os valores 38,$93 ; 19,95 ; 22,43 \mathrm{~g} \mathrm{~kg}^{-1}$ nas mesmas camadas avaliadas neste estudo. Provavelmente os resultados obtidos pelos autores decorrem do maior aporte vegetal, característico do Cerradão, e da textura argilosa do solo, que possui maior interação elétrica com os compostos orgânico do que os solos arenosos.

O valor do C-BMS obtido no PC e SPD na camada de 0,00 a 0,05 m foi 81 e $38 \%$, respectivamente, menor que o observado na área de referência. Na camada de 0,05 a 0,10 m o valor encontrado no PC foi $83 \%$ menor, enquanto o SPD foi $47 \%$ menor que o $\mathrm{CN}$, e na última camada o valor foi $81 \%$ menor no PC e $47 \%$ menor no SPD em comparação com o CN (Tabela 1), evidenciando que os valores do C-BMS no solo sob SPD é maior do que os observados no solo sob PC, pelo menos, duas vezes.

Verifica-se que no solo sob PC, os valores do C-BMS, foram significativamente reduzidos quando comparados com SPD. O aumento do C-BMS decorre da maior quantidade de resíduos culturais na superfície do solo, como reportado por Peres (2010) e Lisboa et al. (2012), que afirmam que a maior quantidade de palhada no solo reduz as perdas de água, favorecendo o desenvolvimento da microbiota do solo (CUNHA et al., 2012; MELO et al., 2017).

Portanto, o SPD promoveu o C-BMS, por outro lado, vale considerar que o C-BMS, embora seja utilizado como indicador da qualidade do solo (FERREIRA et al., 2011); isoladamente este parâmetro não consegue determinar o estado metabólico das comunidades microbianas do solo (BOWLES et al., 2014), evidenciando tão somente uma microfauna desenvolvida e em equilíbrio (FERREIRA et al., 2010; CUNHA et al., 2012; PRAGANA et al., 2012; DUARTE et al., 2014)

Em se tratando da RBS, na camada de 0,00 a 0,05 m, observa-se que solo sob PC se evidenciou $18 \%$ mais ativo que o solo sob CN, contrastando com o solo sob SPD, que foi 34\% menos ativo do que a área de referência. Na camada de 0,05 a 0,10 m o valor da RBS no solo sob PC foi $17 \%$ maior que a referência, enquanto o solo sob SPD foi $33 \%$ menor que o verificado no $C N$ e na camada de 0,10 a 0,20 m a RBS no solo sob PC foi $40 \%$ e no SPD foi $33 \%$ menor do que no $C N$, evidenciando que o solo sob SPD, na camadas superficiais, libera menos $\mathrm{CO}_{2}$ que o PC (Tabela 1 ).

Nas camadas de 0,00 a 0,05 e 0,05 a 0,10 m do solo sob PC, a RBS foi estatisticamente maior do que o observado no solo sob SPD, enquanto na camada de 0,10 a 0,20 não foram observadas diferenças estatísticas significativas entre os tratamentos (Tabela 1). A maior atividade respiratória dos microrganismos, representando maior liberação de $\mathrm{CO}_{2}$ para a atmosfera no solo sob $\mathrm{PC}$, pode estar associado às características intrínsecas do sistema de manejo, que resultam em um ambiente perturbado, corroborando 
os resultados observados neste estudo.

A RBS tende a ser maior em decorrência da atividade microbiana, da decomposição e oxidação de matéria orgânica (VIEIRA et al., 2011; PRAGANA et al., 2012), ademais a RBS depende do estado fisiológico da célula microbiana, que sofre influência da umidade, temperatura, estrutura, disponibilidade de nutrientes, textura do solo, e presença de resíduos orgânicos (SILVA et al., 2010) e principalmente dos sistemas de manejo do solo utilizados.

A mobilização do solo sob PC, tendo como resultado a redução dos teores de COT e C-BMS na camada superficial, contribui para o aumento dos valores de RBS, quando comparado com o SPD, ademais, evidenciase que o maior valor de RBS nas camadas superficiais no solo sob PC relaciona-se ao revolvimento, que acelera o processo de oxidação da MOS, bem como a atividade dos microrganismos, como sugerido por Mazurana et al. (2013) que afirmam que a maior liberação de $\mathrm{CO}_{2}$ ocorre devido à maior atividade biológica, que se relaciona diretamente com o C existente no solo. Sampaio et al. (2008) ao estudarem sistemas de cultivo convencional e indicadores biológicos da qualidade do solo em um Neossolo Quartzarênico no Piauí, verificaram que a adoção de um sistema orgânico aumentou a atividade microbiana e o conteúdo de carbono no solo, corroborando os resultados obtidos neste trabalho. Na Tabela 2 são apresentas as médias e os desvios-padrão do qMic, qCO $\mathrm{CO}_{2}$ e Ds do solo sob PC e SPD em comparação com um Cerrado Nativo (CN) ( $\Delta$ ref, $\%)$.

Tabela 2: Média e desvio-padrão do quociente microbiano ( $q \mathrm{Mic})$, do quociente metabólico $\left(q \mathrm{qO}_{2}\right)$ e da densidade (Ds) de um Latossolo cultivado com Soja sob plantio convencional (PC) e sistema plantio direto (SPD) tendo um Cerrado nativo de fitofisionomia de campo sujo como referência ( $\Delta$ ref, \%) no Oeste da Bahia, Brasil.

\begin{tabular}{|c|c|c|c|c|c|c|}
\hline \multirow[t]{3}{*}{ Tratamento } & qMic & $\Delta$ ref & $\mathrm{qCO}_{2}$ & $\Delta r e f$ & Ds & $\Delta$ ref \\
\hline & $\%$ & $\%$ & $\mathrm{mg} \mathrm{g}^{-1} \mathrm{~h}^{-1}$ & $\%$ & $\mathrm{~g} \mathrm{~cm}^{-3}$ & $\%$ \\
\hline & \multicolumn{6}{|l|}{0,00 a $0,05 \mathrm{~m}$} \\
\hline S_PC & $4,76 \pm 2,16 b$ & -64 & $1.08 \pm 0,35 \mathrm{a}$ & 559 & $1,50 \pm 0,04 a$ & 3 \\
\hline S_SPD & $9,60 \pm 4,85 a$ & -28 & $0.18 \pm 0,02 b$ & 9 & $1,48 \pm 0,08 a$ & 2 \\
\hline \multirow[t]{2}{*}{$\mathrm{CN}$} & 13,37 & 100 & 0.16 & 100 & 1,44 & 100 \\
\hline & \multicolumn{6}{|l|}{0,05 a $0,10 \mathrm{~m}$} \\
\hline S_PC & $4,09 \pm 0,40 \mathrm{~b}$ & -80 & $1.05 \pm 0,26 \mathrm{a}$ & 629 & $1,55 \pm 0,06 b$ & 8 \\
\hline S_SPD & $10,16 \pm 2,76 a$ & -49 & $0.18 \pm 0,04 b$ & 26 & $1,71 \pm 0,14 \mathrm{a}$ & 19 \\
\hline \multirow[t]{2}{*}{$\mathrm{CN}$} & 20,08 & 100 & 0.14 & 100 & 1,43 & 100 \\
\hline & \multicolumn{6}{|l|}{0,10 a $0,20 \mathrm{~m}$} \\
\hline S_PC & $7,05 \pm 1,86 b$ & -66 & $0.41 \pm 0,15 a$ & 220 & $1,58 \pm 0,08 b$ & 7 \\
\hline S_SPD & $11,34 \pm 1,65 a$ & -46 & $0.16 \pm 0,04 b$ & 26 & $1,75 \pm 0,07 a$ & 18 \\
\hline $\mathrm{CN}$ & 20,95 & 100 & 0.13 & 100 & 1,47 & 100 \\
\hline
\end{tabular}

*Soja em Plantio Convencional (S_PC); Soja em consórcio com Crotalária sucedendo Milho, Braquiária e Algodão (S_SPD); Cerrado nativo de campo sujo (CN). Médias seguidas pela mesma letra minúscula nas colunas, dentro de cada parâmetro e camada de solo avaliada não diferem pelo teste de Duncan a 5 \%.

Na camada de 0,00 a 0,05 m do solo sob PC o qMic foi $64 \%$ menor que o valor do $\mathrm{CN}$, enquanto o SPD foi $28 \%$ menor. Na camada de 0,05 a $0,10 \mathrm{~m}$ os valores de qMic observados no solo sob PC e SPD foram 80 e $49 \%$, respectivamente, menores que o observado no $\mathrm{CN}$ e na camada 0,10 a 0,20 m os valores de qMic observados no PC e SPD, foram 66 e 45\% menores, respectivamente, que o valor encontrado na área de referência (Tabela 2), ou seja, os valores de qMic nos tratamentos foram menores que os observados no CN, evidenciando os efeitos dos sistemas de manejo sobre este parâmetro, por outro lado, os valores observado no solo sob SPD foram mais próximos dos observados no $\mathrm{CN}$ do que o valores de qMic no PC. 
Segundo Lisboa et al. (2012), maior qMic se relaciona ao acúmulo de carbono pelos microrganismos, em decorrência da maior disponibilidade de substrato. Assim, valores elevados de qMic permitem afirmar que o solo apresenta condições adequadas para o desenvolvimento microbiano, as quais dependem da adição de matéria orgânica de boa qualidade (JAKELAITIS et al., 2008), como pode ser verificado no solo sob SPD, que nas camadas avaliadas, evidenciou valores distintos e estatisticamente maiores que o PC. Nas camadas três camadas avaliadas, valores de $\mathrm{qCO}_{2}$ observados no solo sob PC foram de 559, 629 e $220 \%$, respectivamente, maiores que o $\mathrm{CN}$, enquanto no solo sob SPD, nas respectivas camadas avaliadas, os valores de $\mathrm{qCO}^{2}$ foram somente 9,26 e $26 \%$ maiores que o solo sob CN (Tabela 2).

Estudos a partir da década de 90 têm mencionado que valores elevados de $\mathrm{qCO}_{2}$ indicam ecossistemas sob condições estressantes ou distúrbios ambientais (BARDGETT et al., 1994; LACERDA et al., 2013), ou seja, caracterizam ambientes em elevado estados de degradação, nesse sentido, diferença estatísticas significativas entre os tratamentos foram constatadas em todas as camadas do solo avaliado, corroborando a afirmativa de que o SPD reduziu os valores de $\mathrm{qCO}_{2}$ e promoveu um ambiente propício ao armazenamento de Carbono, contrastando com o PC. Além disso, diversos autores afirmam que a magnitude de uso e o tipo de manejo do solo aumentam os valores $\mathrm{qCO}_{2}$, como observado no solo sob PC (PRAGANA et al., 2012; LACERDA et al., 2013; DUARTE et al., 2014; GOMES et al., 2015; GUIMARÃES et al., 2017).

A Ds avaliada nos tratamentos, em todas as camadas, foi maior do que a observada no CN (Tabela 2). Na camada de 0,00 a 0,05 m o PC apresentou Ds maior do que o SPD, sem evidenciar diferenças estatísticas, porém, ressalta-se que o uso e manejo do solo, invariavelmente ocasiona alterações importantes nas primeiras camadas do solo (PAULINO et al., 2011). Ademais, nas camadas 0,05 a 0,10 e 0,10 a 0,20 m o SPD apresentou médias de Ds significativamente maiores do que o PC, o que pode ser atribuído ao adensamento natural do solo sob SPD (VASCONCELOS et al., 2014; REIS et al., 2016), devido à ausência de revolvimento.

Tabela 3: Coeficientes de correlação de Pearson entre atributos orgânicos de um Latossolo Vermelho Amarelo distrófico, na camada de 0,00 a 0,20 m, cultivado com Soja sob plantio convencional (PC) e sistema plantio direto (SPD) no Oeste da Bahia, Brasil.

\begin{tabular}{|l|l|l|l|l|l|}
\hline & $\mathrm{C}-\mathrm{BMS}$ & $\mathrm{RBS}$ & $\mathrm{qCO}$ & $\mathrm{qMic}$ & Ds \\
\hline COT & $0,46^{* *}$ & $0,47^{* *}$ & $-0,33^{* *}$ & 0,17 & $-0,02$ \\
\hline C-BMS & & $0,66^{* *}$ & $-0,65^{* *}$ & $0,70^{* *}$ & $0,31^{* *}$ \\
\hline RBS & & & $-0,41^{* *}$ & $0,56^{* *}$ & 0,10 \\
\hline $\mathrm{qCO}_{2}$ & & & & $-0,59^{* *}$ & $-0,35^{* *}$ \\
\hline qMic & & & & & $0,26^{* *}$ \\
\hline
\end{tabular}

**Significativo a $1 \%$ de probabilidade $(\mathrm{n}=24)$. C-BMS: Carbono da Biomassa Microbiana do Solo $\left(\mathrm{mg} \mathrm{C} \mathrm{kg}^{-1}\right)$; RBS: Respiração Basal do Solo $\left(\mu \mathrm{g} \mathrm{kg}^{-1} \mathrm{~h}^{-1}\right)$; $\mathrm{qCO}_{2}$ : Quociente metabólico $\left(\mathrm{mg} \mathrm{g}^{-1} \mathrm{~h}^{-1}\right)$; qMic: Quociente microbiano (\%); Ds: Densidade do solo $\left(\mathrm{g} \mathrm{cm}^{-3}\right)$.

Moraes et al. (2014) e Garrido et al. (2014) relataram incremento da Ds em solo sob SPD, em comparação com solo sob PC. Schwen et al. (2011), ao avaliar o efeito de diferentes sistemas de manejo em um Chernossolo, obtiveram valores médios de Ds significativamente maiores no SPD em comparação ao PC $\left(1,44\right.$ e $\left.1,34 \mathrm{~g} \mathrm{~cm}^{-3}\right)$. Os autores atribuíram essa diferença ao efeito do adensamento natural promovido por 12 anos de adoção do SPD, sem revolvimento do solo, o que também pode ser observado neste trabalho. Na Tabela 3 são apresentados os coeficientes de correlação de Person entre os atributos de um Latossolo 
Vermelho Amarelo distrófico cultivado com soja sob Plantio Convencional e Sistema Plantio Direto no Oeste da Bahia.

O C-BMS $(r=0,46 ; p<0,01)$ e a RBS $(r=0,47 ; p<0,01)$ estiveram positivamente correlacionados com os de COT (Tabela 3), evidenciando a importância da adição de compostos orgânicos na promoção da microbiota do solo e sua atividade. Por outro lado, o $\mathrm{qCO}_{2}(\mathrm{r}=-0,33 ; \mathrm{p}<0,01)$ esteve negativamente correlacionado com o COT, C-BMS $(-0,65 ; p<0,01)$ e RBS $(r=-0,41 ; p<0,01)$, além disso, evidencia-se que o aumento dos valores Ds diminuem o $\mathrm{qCO}_{2}(r=-0,35 ; p<0,01)$ no solo avaliado.

Neste trabalho, verificou-se que o PC apresentou Est_C de $\sim 11 \mathrm{Mg} \mathrm{ha}^{-1}$, o SPD $\sim 17 \mathrm{Mg} \mathrm{ha}^{-1}$ e o CN 15 $\mathrm{Mg} \mathrm{ha}^{-1}$, permitindo ressaltar a capacidade do SPD em armazenar carbono no solo, contrastando com o solo sob PC e com o CN (Figura 1). Reis et al. (2016) avaliando os Est_C em um Planossolo, verificaram que a partir de cinco anos de implantação ( $\left.23 \mathrm{Mg} \mathrm{C} \mathrm{ha}^{-1}\right)$ do SPD, os Est_C passam a apresentar diferenças estatísticas quando comparado com um SPD de um ano ( 13 $\left.\mathrm{Mg} \mathrm{C} \mathrm{ha-1}^{-1}\right)$ de implantação, corroborando os resultados deste trabalho, haja vista que o SPD está implantado há seis anos (Quadro 1).
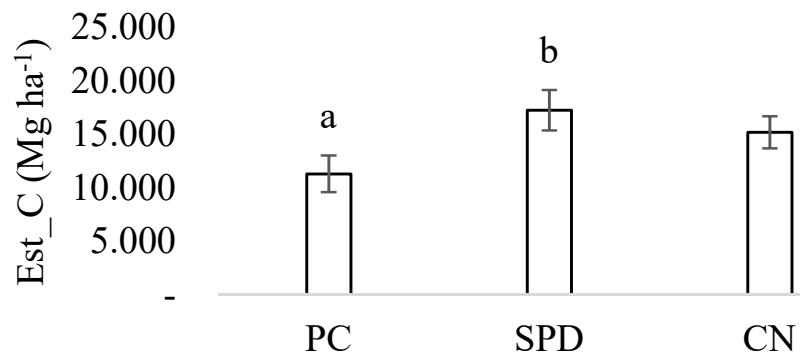

Figura 1: Estoque equivalente de carbono em um Latossolo Vermelho Amarelo distrófico, na camada de 0,00 a 0,20 m, cultivado com Soja sob plantio convencional (PC) e sistema plantio direto (SPD) no Oeste da Bahia, Brasil. Médias seguidas de letras iguais não diferem pelo teste de Duncan a $5 \%$ de probabilidade. Barras verticais indicam os desvios em relação à média.

Martín et al. (2016) avaliando os Est_C na Espanha, verificaram que a camada superficial do solo, no país, possui Est_C médio de 56,57 Mg C ha-1. Sendo observados maiores valores de Est_C em áreas sob floresta $\left(76,34 \mathrm{Mg} \mathrm{Cha}^{-1}\right)$, em áreas de cultivo $\left(41,67 \mathrm{Mg} \mathrm{C}^{-1}\right)$ e em áreas de pastagem $\left(62,49 \mathrm{Mg} \mathrm{Cha}{ }^{-1}\right)$ respectivamente, evidenciando que os solos manejados têm redução nos Est_C.

De maneira geral, os valores de Est_C no solo avaliado são expressivamente inferiores aos apresentados por Martín et al. (2016), o que além de estar relacionado aos sistemas de manejo, podem também estar relacionado ao clima. Entretanto, os valores obtidos neste trabalho aproximam-se dos obtidos por Reis et al. (2016), quem embora tenham realizado as avaliações no Rio Grande do Sul, evidenciam a importância do sistema de manejo para a conservação do Carbono no solo. Loss et al. (2011) afirmam que o maior desenvolvimento do sistema radicular das plantas favorece o conteúdo de MOS, bem como a formação de agregados, que podem favorecer a proteção física da MOS, que consequentemente aumentam os Est_C ao longo do tempo (GUARESCHI et al., 2012).

\section{CONCLUSÕES}

O sistema plantio direto promoveu o carbono orgânico total, a biomassa microbiana do solo, o 
quociente microbiano e os estoques de carbono no solo; A biomassa microbiana do solo e o quociente microbiano são parâmetros sensível para avaliar a qualidade do solo; Solos manejados sob plantio convencional têm redução nos valores de carbono orgânico total, biomassa microbiana do solo, quociente microbiano e nos estoques de carbono no solo, contribuindo para a degradação do solo.

AGRADECIMENTOS: aos apoiadores e financiadores deste trabalho, sobretudo à Coordenação de Aperfeiçoamento de Pessoal de Nível Superior (CAPES), à Fundação de Amparo à Pesquisa do Estado da Bahia (FAPESB); à Fundação de Apoio à Pesquisa e Desenvolvimento do Oeste Baiano (Fundação Bahia); à Empresa Brasileira de Pesquisa Agropecuária (Embrapa) Algodão e Território, bem como a empresa de Fertilizantes JCO.

\section{REFERÊNCIAS}

AIBA. Associação de Agricultores e Irrigantes do Oeste da Bahia. AIBA Rural, v.309, n.4, 2018.

ALVARES, C. A.; STAPE, J. L.; SENTELHAS, P. C.; GONÇALVES, J. L. M.; SPAROVEK, G.. Köppen's climate classification map for Brazil. Meteorologische Zeitschrift, Stuttgart, v.22, n.6, p.711-728, 2013. DOI: http://dx.doi.org/10.1127/09412948/2013/0507

ALVAREZ, C.; ALVAREZ, C. R.; COSTANTINI, A.; BASANTA, M.. Carbon and nitrogen sequestration in soils under different management in the semi-arid Pampa (Argentina). Soil and Tillage Research, v.142, p.25-31, 2014. DOI: http://doi.org/10.1016/j.still.2014.04.005

ALVES, T. D. S.; CAMPOS, L. L.; ELIAS NETO, N.; MATSUOKA, M.; LOUREIRO, M. F.. Biomassa e atividade microbiana de solo sob vegetação nativa e diferentes sistemas de manejos. Acta Scientiarum, Maringá, v.33, n.2, p.341-347, 2011. DOI: http://dx.doi.org/10.4025/actasciagron.v33i2.4841

ANDERSON, T. H.; DOMSCH, K. H.. The metabolic quotient for $\mathrm{CO}_{2}\left(\mathrm{qCO}_{2}\right)$ as a specific activiy parameter to assess the efect of environmental condition, such as $\mathrm{pH}$ on the microbial biomass of forest soils. Soil Biology and Biochemistry, Braunschweig, v.25, n.3, p.393-395, 1993. DOI: http://dx.doi.org/10.1590/01047760201723012228

BARDGETT, R. D.; SAGGAR, S.. Effects of heavy metal contamination on the short-term decomposition of labeled [14C] glucose in a pasture soil. Soil Biology and Biochemistry, Oxford, v.26, p.727-733, 1994. DOI: http://dx.doi.org/10.1016/0038-0717(94)90265-8

BOWLES, T. M.; ACOSTA-MARTÍNEZ, V.; CALDERÓN, F.; JACKSON, L. E.. Soil enzyme activities, microbial communities, and carbon and nitrogen availability in organic agroecosystems across an intensively-managed agricultural landscape. Soil Biology and Biochemistry, v.68, p.252-262, 2014. DOI: http://doi.org/10.1016/j.soilbio.2013.10.004

CARNEIRO, M. A. C.; SOUZA, E. D. D.; REIS, E. F. D.; PEREIRA, H. S.; AZEVEDO, W. R. D.. Atributos físicos, químicos e biológicos de solo de cerrado sob diferentes sistemas de uso e manejo. Revista Brasileira de Ciência do Solo, Viçosa, v.33, p.147-157, 2009. DOI: http://dx.doi.org/10.1590/S0100-

\section{6}

CONAB. Companhia Nacional de Abastecimento. Observatório agrícola: acompanhamento da safra brasileira de grãos: décimo Levantamento. 2019.

CONTI, R.; GUIMARÃES, D. O.; PUPO, M. T.. Aprendendo com as interações da natureza: microrganismos simbiontes como fontes de produtos naturais bioativos. Ciência e Cultura, São Paulo, v.64, n.3, p.43-47, 2012. DOI: http://dx.doi.org/10.21800/S0009-67252012000300014

COSTA JUNIOR, C. C.; PICCOLO, M. C.; NETO, M. S.; CAMARGO, P. B.; CERRI, C. C.; BERNOUX, M.. Carbono total e $\delta 13 C$ em agregados do solo sob vegetação nativa $e$ pastagem no bioma cerrado. Revista Brasileira de Ciências do Solo, Viçosa, v.35, p.1241-1252, 2011. DOI: http://dx.doi.org/10.1590/S0100-06832011000400017

CUNHA, E. Q.; STONE, L. F.; FERREIRA, E. P. B.; DIDONET, A. D.; MOREIRA, J. A. A.. Atributos físicos, químicos e biológicos de solo sob produção orgânica impactados por sistemas de cultivo. Revista Brasileira de Engenharia Agrícola e Ambiental, Campina Grande, v.16, n.1, p.56-63, 2012. DOI: http://dx.doi.org/10.1590/S1415-43662012000100008

DADALTO, J. P.; FERNANDES, H. C.; TEIXEIRA, M. M; CECON, P. R.; MATOS, A. T.. Sistema de preparo do solo e sua influência na atividade microbiana. Revista Engenharia Agricola, Jaboticabal, v.35, n.3, p.506-513, 2015. DOI: http://dx.doi.org/10.1590/1809-4430-Eng.Agric.v35n3p506$513 / 2015$

DUARTE, I. B.; GALLO, A.; GOMES, M.; GUIMARÃES, N.; ROCHA, D.; SILVA, R.. Plantas de cobertura e seus efeitos na biomassa microbiana do solo. Acta Iguazu, Cascavel, v.3, n.2, p.150-165, 2014.

ELEFTHERIADIS, A.; TURRIÓN M. B.. Soil microbiological properties affected by land use, management, and time since deforestations and crop establishment. European Journal of Soil Biology, v.62, p.138-144, 2014. DOI: http://dx.doi.org/10.1016/j.ejsobi.2014.03.001

FERREIRA, E. P. B.; SANTOS, H. P.; COSTA, J. R.; DE-POLLI, H.; RUMJANEK, N. G.. Microbial soil quality indicators under 
different crop rotations and tillage management. Revista Ciência Agronômica, Fortaleza, v.41, n.2, p.177-183, 2010. DOI: http://dx.doi.org/10.1590/S1806-66902010000200002

FERREIRA, E. P. B.; WENDLAND, A.; DIDONET, A. D. Microbial biomass and enzyme activity of a Cerrado Oxisol under agroecological production system. Bragantia, Campinas, v.70, n.4, p.1-9, 2011. DOI: http://dx.doi.org/10.1590/S0006-87052011000400024

FREITAS, L.; OLIVEIRA, I. A.; CASAGRANDE, J. C.; SILVA, L. S.; CAMPOS, M. C. C.. Estoque de carbono de latossolos em sistemas de manejo natural e alterado. Ciências Florestais, Santa Maria, v.28, n.1, p.228-239, 2018. DOI: http://dx.doi.org/10.5902/1980509831575

GARRIDO, R.; MADEJÓN, E.; LEÓN-CAMACHO, M.; GIRÓN, I.; MORENO, F.; MURILLO, J. M.. Reduced tillage as an alternative to no-tillage under Mediterranean conditions: a case study. Soil and Tillage Research, v.140, p.40-47, 2014. DOI: http://dx.doi.org/10.1016/j.still.2014.02.008

GAZZOLA, P. R.; GUARESCHI, R. F.; PERIN, A.; PEREIRA, M. G.; ROSSI, C. Q.. Frações da matéria orgânica do solo sob pastagem, sistema plantio direto e integração lavourapecuária. Semina: Ciências Agrárias, Londrina, v.36, n.2, p.693-704, 2015. DOI: http://dx.doi.org/10.5433/16790359.2015v36n2p693

GOMES, S. S.; GOMES, M. S.; GALLO, A. S.; MERCANTE, F. M.; BATISTOTE, M.; SILVA, R. F.. Bioindicadores de qualidade do solo cultivado com milho em sucessão a adubos verdes sob bases agroecológicas. Revista de La Facultad de Agronomia, v.114, n.3, p.30-37, 2015.

GUARESCHI, R. F.; PEREIRA, M. G.; PERIN, A.. Deposição de resíduos vegetais, matéria orgânica leve, estoques de Carbono e Nitrogênio e Fósforo remanescente sob diferentes sistemas de manejo no cerrado Goiano. Revista Brasileira de Ciência do Solo, Viçosa, v.36, n.3, p.909-920, 2012. DOI: http://dx.doi.org/10.1590/S0100$\underline{06832012000300021}$

GUIMARÃES, N. F.; GALLO, A. S.; FONTANETTI, A.; MENEGHIN, S. P.; SOUZA, M. D.B.; MORINIGO, K. P. G.; SILVA, R. F.. Biomassa e atividade microbiana do solo em diferentes sistemas de cultivo do cafeeiro. Revista de Ciências Agrárias, v.40, n.1, p.34-44, 2017. DOI: http://dx.doi.org/10.19084/RCA16041

HOUGHTON, J. T.; DING, Y.; GRIGGS, D. J.; NOGUER, M.; VAN DER LINDEN, P. J.; DAI, X.; MASKELL, K.; JOHNSON, C. A.. Climate Change 2001: The Scientific Basis. Cambridge University Press, Cambridge, v.22, p.1144-1144, 2001. DOI: http://dx.doi.org/10.1002/joc.763

INSAM, H.; DOMSCH, K. H.. Relationship between soil organic carbon and microbial biomass on chronosequences of reclamation sites. Microbial Ecology, New York, v.15, n.2, p.177-188, 1988. DOI: http://dx.doi.org/10.1007/BF02011711

JENKINSON, D. S.; POWLSON, D. S.. The effects of biocidal treatments on metabolism in soil. V. A method for measuring soil biomass. Soil Biology and Biochemistry, Oxford, v.8, n.3, p.209-213, 1976. DOI: http://doi.org/10.1016/0038-0717(76)90005-5
KOPITTKE, P. M.; DALAL, R. C.; FINN, D.; MENZIES, N. W. Global changes in soil stocks of carbon, nitrogen, phosphorus, and sulphur as influenced by long-term agricultural production. Global change biology, v. 23, p.2509-2519, 2017. DOI:

http://dx.doi.org/10.1111/gcb.13513

LACERDA, K. A. P.; CORDEIRO, M. A. S.; VERGINASSI, A.; SALGADO, F. H. M. PAULINO, H. B.; CARNEIRO, M. A. C.. Organic carbon, biomass and microbial activity in an Oxisol under different management systems. Amazonian Journal of Agricultural and Environmental Sciences, v.56, n.3, p.249-254, 2013. DOI: http://dx.doi.org/10.4322/rca.2013.036

LEPSCH, I. F.. Formação e conservação dos solos. 2 ed. São Paulo: Oficina de Textos, 2010.

LISBOA, B. B.; VARGAS, L. K.; SILVEIRA, A. O. D.; MARTINS, A. F.; SELBACH, P. A.. Indicadores microbianos de qualidade do solo em diferentes sistemas de manejo. Revista Brasileira de Ciência do Solo, Viçosa, v.36, n.1, p.33-44, 2012. DOI: http://dx.doi.org/10.1590/S0100-06832012000100004

LOSS, A.; PEREIRA, M. G.; GIACOMO, S. G.; PERIN, A.; ANJOS, L. H. C.. Agregação, carbono e nitrogênio em agregados do solo sob plantio direto com integração lavoura-pecuária. Pesquisa Agropecuária Brasileira, Brasília, v.46, n.10, p.1262-1276, 2011. DOI: http://dx.doi.org/10.1590/S0100204X2011001000022

LOURENTE, E. R. P.; MERCANTE, F. M.; ALOVISI , A. M. T.; GOMES, C. F.; GASPARINI, A. S.; NUNES, C. M.. Atributos microbiológicos, químicos e físicos de solo sob diferentes sistemas de manejo e condições de cerrado. Pesquisa Agropecuária Tropical, Goiânia, v.41, n.1, p.20-28, 2011. DOI: http://dx.doi.org/10.5216/pat.v41i1.8459

MALHEIROS, R.. A influência da sazonalidade na dinâmica da vida no Bioma Cerrado. Revista Brasileira de Climatologia, Curitiba, v.19, p.113-128, 2016. DOI: http://dx.doi.org/10.5380/abclima.v19i0.48876

MARTÍN, J. A. R.; ÁLVARO-FUENTES, J.; GONZALO, J.; GIL, C.; RAMOS-MIRAS, J. J.; GRAU CORBÍ, J. M.; BOLUDA, R.. Assessment of the soil organic carbon stock in Spain. Geoderma, v.264, p.117-125, 2016. DOI: http://dx.doi.org/10.1016/i.geoderma.2015.10.010

MAZURANA, M.; FINK, J. R.; CAMARGO, E.; SCHNITT, R. A.; CAMARGO, F. A. O.. Estoque de carbono e atividade microbiana em sistema de plantio direto consolidado no Sul do Brasil. Revista de Ciências Agrárias, Lisboa, v.36, n.3, p.288-296, 2013

MELO, V. F.; SILVA, D. T.; EVALD, A.; ROCHA, P. R. R. Qualidade química e biológica do solo em diferentes sistemas de uso em ambiente de savana. Revista Agro@mbiente On-line, Boa Vista, v.11, n.2, p.101-110, 2017. DOI: http://dx.doi.org/10.18227/19828470ragro.v11i2.3850

MAPA. Ministério da Agricultura, Pecuária e Abastecimento Secretaria de Política Agrícola. Brasil projeções do agronegócio 2016/2017 a 2026/2027. Brasília: MAPA, 2017. 
MORAES, M. T.; DEBIASI, H.; CARLESSO, R.; FRANCHINI, J. C.; SILVA, V. R.. Critical limits of soil penetration resistance in a rhodic Eutrudox. Revista Brasileira de Ciência do Solo, Viçosa, v.38, p.288-298, 2014. DOI: http://dx.doi.org/10.1590/S0100-06832014000100029

NUNES, M. R.; DENARDIN, J. E.; FAGANELLO, A.; PAULETTO, E.A.; PINTO, L. F. S.. Efeito de semeadora com haste sulcadora para ação profunda em solo manejado com plantio direto. Revista Brasileira de Ciência do Solo, Viçosa, v.38, p.627-638, 2014. DOI:

http://dx.doi.org/10.1590/S0100-06832014000200027

PAULINO, J.; ZOLIN, C. A.; BERTONHA, A.; FREITAS, P. S. L.; FOLEGATTI, M. V.. Estudo exploratório do uso de vinhaça ao longo do tempo. II Caracterísiticas da cana-de-açúcar. Revista Brasileira de Engenharia Agrícola e Ambiental, Campina Grande, v.15, n.3, p.244-249, 2011. DOI: http://dx.doi.org/10.1590/S1415-43662011000300004

PERES, J. G.; SOUZA, C. F.; LAVORENTI, N. A.. Avaliação dos efeitos da cobertura de palha de cana de açúcar na umidade e na perda de água no solo. Engenharia Agrícola, Jaboticabal, v.30, n.5, p.875-886 2010. DOI: http://dx.doi.org/10.1590/S0100-69162010000500010

POLLI, H.; PIMENTEL, M. S.. Indicadores de qualidade do solo. In: AQUINO, A. M.; ASSIS, R. L.. Processos biológicos no sistema solo-planta: ferramentas para uma agricultura sustentável. Brasília: Embrapa, 2005.

POLLI, H.; GUERRA, J. G. M.. Carbono, nitrogênio e fósforo da biomassa microbiana do solo. 2. ed. In: SANTOS, G. A.; SILVA, L. S.; CANELLAS, L. P.; CAMARGO, F. A. O.. Fundamentos da matéria orgânica do solo: ecossistemas tropicais \& subtropicais. Porto Alegre: Metrópole, 2008.

PRAGANA, R. B.; NÓBREGA, R. S. A.; RIBEIRO, M. R.; LUSTOSA FILHO, J. F.. Atributos biológicos e dinâmica da matéria orgânica em Latossolos Amarelos na região do Cerrado piauiense sob sistema plantio direto. Revista Brasileira de Ciência do Solo, Viçosa, v.36, n.3, p.851-858, 2012. DOI: http://dx.doi.org/10.1590/S010006832012000300015

REIS, D. A.; LIMA, C. L. R.; BAMBERG, A. L.. Developing a Soil Physical Quality Index (SPQi) for lowlands under different deployment times of no-tillage. Scientia Agricola, Piracicaba, v.76, n.2, p.157-164, 2019. DOI: http://dx.doi.org/10.1590/1678-992x-2017-0196

REIS, D. A.; LIMA, C. L. R.; BAMBERG, A. L.. Qualidade física e frações da matéria orgânica de um Planossolo sob sistema plantio direto. Pesquisa Agropecuária Brasileira, Brasília, v.51, n.9, p.1623-1632, 2016. DOI: http://dx.doi.org/10.1590/S0100-204X2016000900062

RESENDE, M.; CURI, N.; REZENDE, S. B.; CORRÊA, G. F.; KER, J. C.. Pedologia: Base para distinção de Ambientes. 6 ed. Lavras: UFLA, 2014.

ROMERO, M. L. F.; PARRAS-ALCÁNTARA, L.; LOZANOGARCÍA, B.; CLARK, J. M.; COLLINS, C. D.. Soil quality assessment based on carbon stratification index in different olive grove management practices in Mediterranean áreas. Catena, v.137, p.449-458, 2016. DOI: http://doi.org/10.1016/i.catena.2015.10.019
ROSSI, C. Q.; PEREIRA, M. G.; GIACOMO, S. G.; BETTA, M.; POLIDORO, J. C.. Frações húmicas da matéria orgânica do solo cultivado com soja sobre palhada de braquiária e sorgo. Bragantia, Campinas, v.70, n.3, p.622-630, 2011. DOI: http://dx.doi.org/10.1590/S0006-87052011000300018

SALTON, J. C.; MIELNICZUK, J.; BAYER, C.; FABRÍCIO, A. C.; MACEDO, M. C. M.; BROCH, D. L.. Teor e dinâmica do carbono no solo em sistemas de integração lavourapecuária, Dourados, MT. Pesquisa Agropecuária Brasileira, Brasília, v.46, n.10, p.349-1356, 2011. DOI: http://dx.doi.org/10.1590/S0100-204X2011001000031

SAMPAIO, D. B.; ARAÚJO, A. S. F.; SANTOS, V. B.. Avaliação de indicadores biológicos de qualidade do solo sob sistemas de cultivo convencional e orgânico de frutas. Ciência e Agrotecnologia, Lavras, v.32, n.2, p.353-359, 2008. DOI: http://dx.doi.org/10.1590/S1413-70542008000200001

SCHWEN, A.; BODNER, G.; SCHOLL, P.; BUCHAN, G. D.; LOISKANDL, W.. Temporal dynamics of soil hydraulic Properties and the water-conducting porosity under different tillage. Soil and Tillage Research, v.113, p.89-98, 2011. DOI: http://dx.doi.org/10.1016/j.still.2011.02.005

SEGUY, L.; BOUZINAC, S.. Alternativas de plantio direto de alta performance. In: BELOT, J. L.. Manual de boas práticas de manejo do algodoeiro em Mato Grosso. Cuiabá: IMAmt, 2012. p.38-43.

SILVA JÚNIOR, J.. Potencial de sequestro de Carbono em solos do Cerrado. In: Bases Sustentáveis do Agronegócio. Composer, 2016

SILVA, E. E.; AZEVEDO, P. H. S.; POLLI, H.. Determinação do carbono da biomassa microbiana do solo (BMS-C). Seropédica: Embrapa Agrobiologia, 2007.

SILVA, E. E.; AZEVEDO, P. H. S.; POLLI, H.. Determinação da respiração basal (RBS) e quociente metabólico do solo $\left(q \mathrm{CO}_{2}\right)$. Seropédica: Embrapa Agrobiologia, 2007.

SILVA, R. R. D.; SILVA, M. L. N.; CARDOSO, E. L.; MOREIRA, F. M. D. S.; CURI, N.; ALIVISI, A. M. T.. Biomassa e atividade microbiana em solos sob diferentes sistemas de manejo na região fisiográfica campos das vertentes - MG. Revista Brasileira de Ciência do Solo, Viçosa, v.34, n.5, p.1584-1592, 2010. DOI: http://dx.doi.org/10.1590/S0100$\underline{06832010000500011}$

SISTI, C. P. J.; SANTOS, H. P.; KOHHANN, R.; ALVES, B. J. R.; SEGUNDO, U.; BODDEY, R. M.. Change in carbon and nitrogen stocks in soil under 13 years of conventional or zero tillage in southern Brazil. Soil and Tillage Research, v.76, p.39-58, 2004. DOI: http://dx.doi.org/10.1016/i.still.2003.08.007

SAS. Statistical Analysis System Institute. Procedure guide for personal computers: version 9. Cary: SAS, 1999.

TANG, S.; GUO, J., SHUCHENG, L, J.; XIE, S.; ZHAI, X.; WANG, C.; ZHANG, Y.; WANG, K.. Synthesis of soil carbon losses in response to conversion of grassland to agriculture land. Soil and Tillage Research, v.185, p.29-35, 2019. DOI: http://dx.doi.org/10.1016/j.still.2018.08.011 
TEIXEIRA, P. C.; DONAGEMMA, G. K.; FONTANA, A.; TEIXEIRA, W. G.. Manual de métodos de análise de solo. 3 ed. Brasília: Embrapa, 2017.

VANCE, E. D.; BROOKES, P. C.; JENKINSON, D. S.. An extraction method for measuring soil microbial biomass-C. Soil Biology and Biochemistry, v.19, n.6, p.703-707, 1987. DOI: http://dx.doi.org/10.1016/0038-0717(87)90052-6

VASCONCELOS, R. F. B.; SOUZA, E. R.; CANTALICE, J. R. B.; SILVA, L. S.. Qualidade física de um Latossolo Amarelo de tabuleiros costeiros em diferentes sistemas de manejo da cana-deaçúcar. Revista Brasileira de Engenharia Agrícola e Ambiental, Campina Grande, v.18, n.4, p.381-386, 2014. DOI: http://dx.doi.org/10.1590/S1415-43662014000400004

VELDKAMP, E.. Organic carbon turnover in three tropical soils under pasture after deforestation. Soil Science Society of American Journal, v.58, p.175-180, 1994. DOI: http://dx.doi.org/10.2136/sssaj1994.0361599500580001002 $\underline{5 x}$

VIEIRA, G. D.; CASTILHOS, D. D.; CASTILHOS, R. M. V. Atributos microbianos do solo após a adição de lodo anaeróbio da estação de tratamento de efluentes de parboilização do arroz. Revista Brasileira de Ciência do Solo, Viçosa, v.35, n.2, p.543-550, 2011. DOI: http://dx.doi.org/10.1590/S0100-06832011000200023

ZHOU, H.; ZHANG, D.; WANG, P.; LIU, X.; CHENG, K.; LI, L.; ZHENG, J.; ZHANG, X.; ZHENG, J.; CROWLEY, D.; ZWIETEN, L.; PAN, G.. Changes in microbial biomass and the metabolic quotient with Biochar addition to agricultural soils: A Metaanalysis. Agriculture. Ecosystems and Environment, v.239, p.80-89, 2017. DOI:

http://doi.org/10.1016/i.agee.2017.01.006

A CBPC - Companhia Brasileira de Produção Científica (CNPJ: 11.221.422/0001-03) detém os direitos materiais desta publicação. Os direitos referem-se à publicação do trabalho em qualquer parte do mundo, incluindo os direitos às renovações, expansões e disseminações da contribuição, bem como outros direitos subsidiários. Todos os trabalhos publicados eletronicamente poderão posteriormente ser publicados em coletâneas impressas sob coordenação da Sustenere Publishing, da Companhia Brasileira de Produção Científica e seus parceiros autorizados. Os (as) autores (as) preservam os direitos autorais, mas não têm permissão para a publicação da contribuição em outro meio, impresso ou digital, em português ou em tradução. 